High-resolution gamma ray spectroscopy measurements of the fast ion energy distribution in JET ${ }^{4}$ He plasmas

This article has been downloaded from IOPscience. Please scroll down to see the full text article.

2012 Nucl. Fusion 52063009

(http://iopscience.iop.org/0029-5515/52/6/063009)

View the table of contents for this issue, or go to the journal homepage for more

Download details:

IP Address: 128.178.125.223

The article was downloaded on 28/02/2013 at $15: 16$

Please note that terms and conditions apply. 


\title{
High-resolution gamma ray spectroscopy measurements of the fast ion energy distribution in JET ${ }^{4} \mathrm{He}$ plasmas
}

\author{
M. Nocente ${ }^{1,2}$, M. Tardocchi ${ }^{2}$, V.G. Kiptily ${ }^{3}$, P. Blanchard ${ }^{3}$, \\ I. Chugunov ${ }^{4}$, S. Conroy ${ }^{5}$, T. Edlington ${ }^{3}$, A.M. Fernandes ${ }^{6}$, \\ G. Ericsson ${ }^{5}$, M. Gatu Johnson ${ }^{5}$, D. Gin ${ }^{4}$, G. Grosso ${ }^{2}$, \\ C. Hellesen ${ }^{5}$, K. Kneupner ${ }^{3}$, E. Lerche ${ }^{7}$, A. Murari ${ }^{8}$, A. Neto ${ }^{6}$, \\ R.C. Pereira ${ }^{6}$, E. Perelli Cippo ${ }^{1}$, S. Sharapov ${ }^{3}$, A. Shevelev ${ }^{4}$, \\ J. Sousa ${ }^{6}$, D.B. Syme ${ }^{3}$, D. Van Eester ${ }^{7}$, G. Gorini ${ }^{1,2}$ and \\ JET-EFDA Contributors ${ }^{\mathrm{a}}$
}

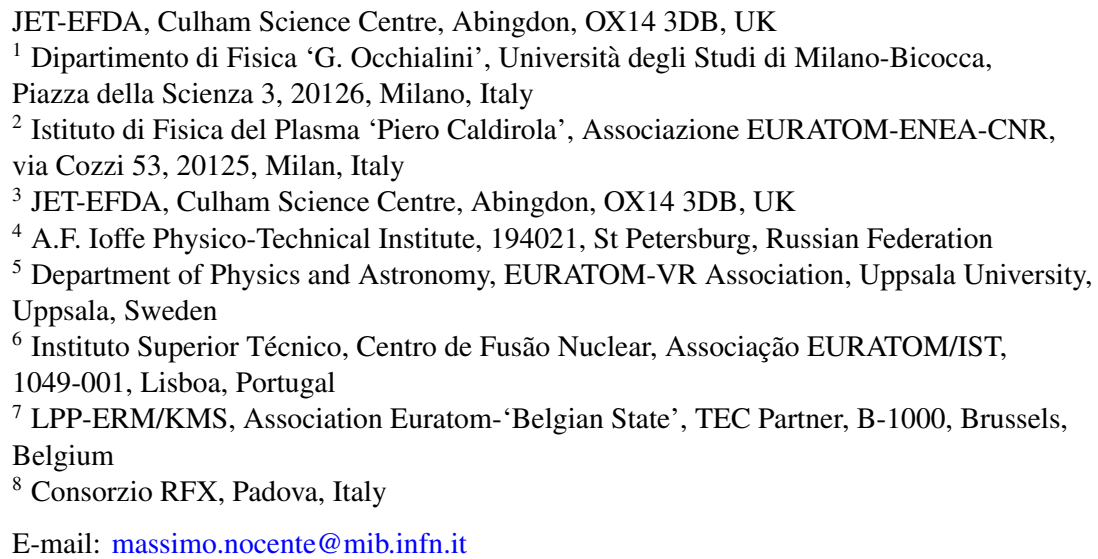

Received 9 January 2012, accepted for publication 23 March 2012

Published 24 April 2012

Online at stacks.iop.org/NF/52/063009

\begin{abstract}
High-resolution $\gamma$-ray measurements were carried out on the Joint European Torus (JET) in an experiment aimed at accelerating ${ }^{4} \mathrm{He}$ ions in the $\mathrm{MeV}$ range by coupling third harmonic radio frequency heating to an injected ${ }^{4} \mathrm{He}$ beam. For the first time, Doppler broadening of $\gamma$-ray peaks from the ${ }^{12} \mathrm{C}(\mathrm{d}, \mathrm{p} \gamma){ }^{13} \mathrm{C}$ and ${ }^{9} \mathrm{Be}(\alpha, \mathrm{n} \gamma){ }^{12} \mathrm{C}$ reactions was observed and interpreted with dedicated Monte Carlo codes based on the detailed nuclear physics of the processes. Information on the confined ${ }^{4} \mathrm{He}$ and deuteron energy distribution was inferred, and confined ${ }^{4} \mathrm{He}$ ions with energies as high as $6 \mathrm{MeV}$ were assessed. A signature of MHD activity in $\gamma$-ray traces was also detected. The reported results have a bearing on diagnostics for fast ions in the $\mathrm{MeV}$ range in next step fusion devices.
\end{abstract}

(Some figures may appear in colour only in the online journal)

\section{Introduction}

Understanding the behaviour of fast ions is still widely considered an open problem for reliable operation of a fusion device. There has been remarkable progress in the past decade in this field of research, both on the theoretical as well as on the experimental side, as recently reviewed in [1]. Many of the

a See the appendix of Romanelli F. et al 2010 Proc. 23rd IAEA Fusion Energy Conf. 2010 (Daejeon, Korea, 2010) http://www-pub.iaea.org/mtcd/ meetings/PDFplus/2010/cn180/cn180_papers/ov_1-3.pdf. predicted magnetohydrodynamic (MHD) modes driven by fast particles have been identified and their non-linear frequency evolution has been measured and theoretically explained. A certain degree of uncertainty, however, remains on the effects of the interaction between these modes and fast ions which has a bearing on $\alpha$ particles in a fusion reactor. Fast ion redistribution and losses due to the excitation of many MHD instabilities, in addition to the consequent role of energetic particles in the overall transport, are largely unknown and dedicated experiments are currently carried out in several 
machines [1]. On the diagnostics side, most of the current knowledge derives from measurements of fast ions on middlesized machines, where instabilities are driven by particles with energies in the $100 \mathrm{keV}$ range, either due to neutral beam injection (NBI) or generated by radio frequency (RF) heating. Recent progress in the detection of lost ions allowed phase space characterization of the losses [2,3], while a charge exchange recombination spectroscopy technique based on fast ion $\mathrm{D}_{\alpha}$ emission (FIDA) [4] showed flattening of the fast ion profile correlated with the onset of energetic particle instabilities $[5,6]$. As the energy of the ions is increased towards the $\mathrm{MeV}$ range, such as that of $\alpha$ particles in a burning plasma, many diagnostics currently used for these studies on middle-sized machines show limitations and new methods need to be sought. For example, the considerably small cross sections for $\mathrm{D}_{\alpha}$ emission in the $\mathrm{MeV}$ range limit the applicability of the FIDA technique [4]. On the other hand, the increased size of next step devices demands methods to diagnose confined energetic ions and complement information on losses.

Measurements of confined energetic ions in large volume, next step tokamaks, such as ITER, are considered a challenging task [7]. Apart from the interest in studying fast ion driven MHD modes, diagnosis of confined fast ions is needed to assess the production and slowing down of $\alpha$ particles, or to evaluate the efficiency of certain RF heating schemes on ITER. In addition to $\gamma$-ray measurements, the present set of diagnostics envisaged for confined energetic ions on ITER comprises collective Thomson scattering (CTS), charge exchange recombination spectroscopy (CXRS) and knock-on measurements [7]. All these diagnostic techniques present some limitations. In particular, it is still unclear whether the signal-to-noise ratio will be sufficient for CXRS and CTS operations. Knock-on measurements, which are based on the detection of fast deuterons and tritons produced from nuclear elastic scattering of fast ions, were demonstrated at JET using neutron spectroscopy [8] and neutral particle analysers [9]. These, however, are an indirect measurement of confined energetic ions.

Gamma ray spectroscopy is the proposed technique for direct observation of ions in the $\mathrm{MeV}$ range in next step fusion devices and a number of studies have been made on the JET tokamak, the only machine that can confine ions in that energy range [10]. In recent years, the upgrade of the JET $\gamma$-ray spectrometers with the installation of a high-purity germanium ( $\mathrm{HpGe}$ ) and a lanthanum bromidecerium $\left(\mathrm{LaBr}_{3}(\mathrm{Ce})\right)$ detector [11] has considerably enhanced the quality of observations and the information that can be derived from the data. For the first time, the Dopplerbroadened peak shape produced by the interaction of ${ }^{3} \mathrm{He}$ ions with ${ }^{12} \mathrm{C}$ impurities has been measured and interpreted in terms of the nuclear physics of the reaction and tail temperature of the energetic ions [12]. In this paper we report, for the first time, observations of Doppler-broadened peak shapes from energetic ${ }^{4} \mathrm{He}$ ions and deuterons on JET. The measurements were carried out in an experiment aimed at accelerating ${ }^{4} \mathrm{He}$ ions by coupling RF heating at the third harmonic to a helium beam injected approximately at $40^{\circ}$ to the magnetic axis in a (D) ${ }^{4} \mathrm{He}$ plasma [13]. The plasma current was $I_{\mathrm{p}}=2 \mathrm{MA}$ and the toroidal magnetic field at the plasma centre was $B_{\mathrm{T}}=$

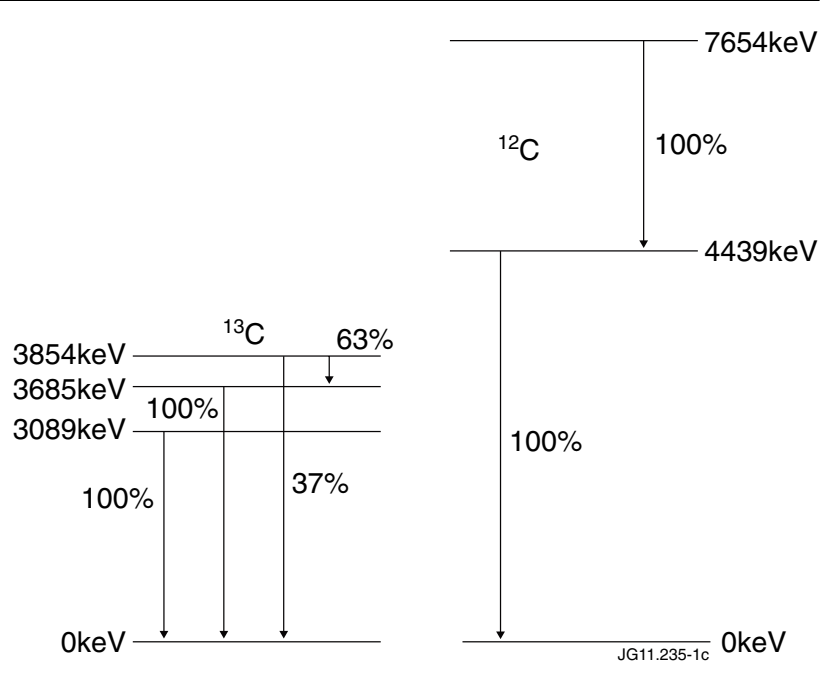

Figure 1. Level scheme of the ${ }^{13} \mathrm{C}$ and ${ }^{12} \mathrm{C} \gamma$ emitting nuclei produced in the ${ }^{12} \mathrm{C}(\mathrm{d}, \mathrm{p} \gamma){ }^{13} \mathrm{C}$ and ${ }^{9} \mathrm{Be}(\alpha, \mathrm{n} \gamma){ }^{12} \mathrm{C}$ reactions.

2.25 $\mathrm{T}$, which placed the $51.4 \mathrm{MHz}$ resonance layer on the magnetic axis for both ${ }^{4} \mathrm{He}$ and $\mathrm{d}$ ions. Core electron densities in the range $(2.5-4) \times 10^{19} \mathrm{~m}^{-3}$ with average on-axis electron temperatures of $3.4 \mathrm{keV}$ were available for applied antenna powers in the range 3-6 MW. The collected experimental data are interpreted in this paper by means of simulations with the GENESIS (Gamma Emission and Neutron Emission Simulation and Interpretation Software) Monte Carlo code that is an extended version of a previous program $[14,15]$ adopted for neutron emission calculations. A simplified semi-analytical model, described in section 3, is adopted for the distribution of RF-heated ${ }^{4} \mathrm{He}$ and $\mathrm{d}$ ions as input for calculations. This simplification was found convenient to numerically investigate changes in the peak shapes and ratios with respect to modifications in parameters describing the energetic ion distribution, while retaining the main features of the employed heating scheme. After a short overview of the $\gamma$ emitting reactions of interest for this study in section 2, the experimental results and simulations are presented, and the findings compared with information from other diagnostics. Implications of these results on observations of confined fast ions in the $\mathrm{MeV}$ range on next generation devices are finally discussed.

\section{Gamma-emitting reactions and cross sections}

Gamma ray emission can occur when fast ions interact with impurities, either coming from the machine vacuum vessel or injected on purpose. On JET there are two reactions of interest for the observation of fast ${ }^{4} \mathrm{He}$ ions and deuterons, the ${ }^{9} \mathrm{Be}(\alpha, \mathrm{n} \gamma){ }^{12} \mathrm{C}$ and ${ }^{12} \mathrm{C}(\mathrm{d}, \mathrm{p} \gamma){ }^{13} \mathrm{C}$ reactions, respectively [16]. The level scheme of the emitting nuclei is presented in figure 1. The ${ }^{9} \mathrm{Be}(\alpha, \mathrm{n} \gamma){ }^{12} \mathrm{C}$ reaction is the main candidate for $\alpha$ particle observations through gamma ray spectrometry on ITER $[17,18]$ and has a positive $Q$ value of $1.27 \mathrm{MeV}$ for populating ${ }^{12} \mathrm{C}$ in its first excited state, which in turn de-excites by emission of a $4.44 \mathrm{MeV}$ gamma ray. If the $\alpha$ particle energy exceeds $E_{\alpha}=1.35 \mathrm{MeV}$ the second ${ }^{12} \mathrm{C}$ excited state starts to be populated and de-excites with the emission of 3.22 and 4.44 MeV gamma rays in cascade. The ${ }^{12} \mathrm{C}(\mathrm{d}, \mathrm{p} \gamma){ }^{13} \mathrm{C}$ reaction 


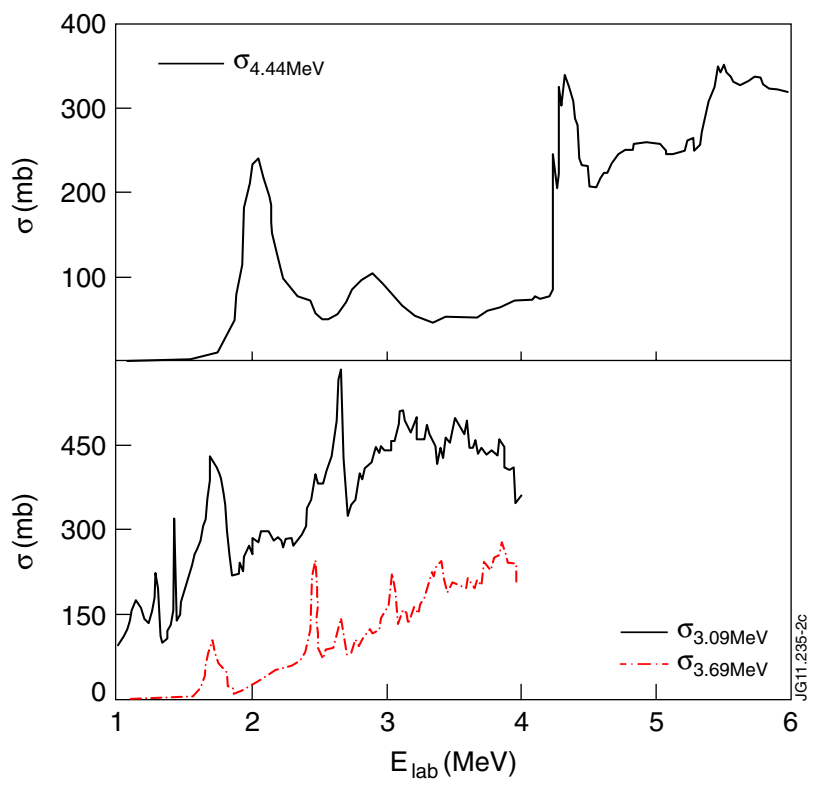

Figure 2. Cross section for the production of $\gamma$-rays at energies $E_{\gamma}=4.44 \mathrm{MeV}$ (top, ${ }^{9} \mathrm{Be}(\alpha, \mathrm{n} \gamma){ }^{12} \mathrm{C}$ reaction), $E_{\gamma}=3.09 \mathrm{MeV}$ and $3.69 \mathrm{MeV}$ (bottom, ${ }^{12} \mathrm{C}(\mathrm{d}, \mathrm{p} \gamma){ }^{13} \mathrm{C}$ reaction) as a function of the light ion energy in the laboratory frame. The values shown are weighted by the branching ratios of figure 1 .

is instead endothermic, with negative $Q$ values of $-0.37 \mathrm{MeV}$, $-0.96 \mathrm{MeV}$ and $-1.13 \mathrm{MeV}$ for the population of the first, second and third ${ }^{13} \mathrm{C}$ excited states, respectively, yielding gamma rays of energies $3.09 \mathrm{MeV}, 3.68 \mathrm{MeV}$ and $3.85 \mathrm{MeV}$. In the discharges analysed in this paper ${ }^{9} \mathrm{Be}$ was evaporated overnight while ${ }^{12} \mathrm{C}$ was present at a typical concentration level of about $1 \%$ due to the composition of the JET vacuum vessel.

The cross sections for the production of gamma rays observed during the measurements reported here are shown in figure 2 , and were obtained by merging experimental data from several authors in a way similar to the methods described in [19]. In particular, data from [20,21] were used to obtain the total, as well as differential, cross section for production of the $4.44 \mathrm{MeV}$ peak from the ${ }^{9} \mathrm{Be}(\alpha, \mathrm{n} \gamma){ }^{12} \mathrm{C}$ reaction up to $E_{\alpha}=6 \mathrm{MeV}$ (figure 2, top). The cross section is negligible below $E_{\alpha}<1 \mathrm{MeV}$ and displays a non-trivial behaviour as a function of energy with major resonances at $E_{\alpha} \approx 2.0,2.9$, 4.3, 5.5 MeV. The angular distribution of the emitted neutrons in the c.m. frame is not isotropic and shows an increased forward peaking as the energy of ${ }^{4} \mathrm{He}$ ions is raised [20,21], which affects the Monte Carlo simulated peak shape.

Cross section data for the ${ }^{12} \mathrm{C}(\mathrm{d}, \mathrm{p} \gamma){ }^{13} \mathrm{C}$ reaction were obtained from [22], and are shown in figure 2, bottom part, for laboratory energies in the range $1-4 \mathrm{MeV}$. The values are those for the production of 3.09 and $3.69 \mathrm{MeV}$ gamma rays, which are weighted by the branching ratios of figure 1. Similarly to the $\alpha+{ }^{9} \mathrm{Be}$ reaction, the cross section for the $\mathrm{d}+{ }^{12} \mathrm{C}$ reaction has a complex trend as a function of energy, with several resonances appearing in the $\mathrm{MeV}$ region.

\section{Fast ion energy distribution}

For energies far from the thermal ion region, an approximate form for the anisotropic fast ${ }^{4} \mathrm{He}$ energy distribution $f$ is obtained from the Fokker-Planck equation

$$
\frac{\partial f}{\partial t}+S\left(v_{\mathrm{p}}\right)+L\left(v_{\mathrm{p}}\right)=C(f)+Q(f)
$$

where all the quantities are expressed in terms of the velocity component perpendicular to the magnetic field $v_{\mathrm{p}}$ only. $S\left(v_{\mathrm{p}}\right)$ represents the source term due to perpendicular NBI injection and is expressed as $S\left(v_{\mathrm{p}}\right)=S_{0} /\left(2 \pi v_{\mathrm{p}}\right) \cdot \delta\left(v_{\mathrm{p}}-v_{0}\right)$ with $v_{0}$ indicating the NBI injection velocity and $S_{0}$ a constant. $L\left(v_{\mathrm{p}}\right)$ is a loss term that absorbs ions at the same rate as $S\left(v_{\mathrm{p}}\right)$, but at thermal speed. $C(f)$ and $Q(f)$ are operators representing the effect of collisions and RF heating on the alpha particles, respectively. Following the treatment of [23] they can be expressed as

$$
\begin{aligned}
& C(f)+Q(f)=\frac{1}{v_{\mathrm{p}}} \frac{\partial}{\partial v_{\mathrm{p}}}\left[-\alpha v_{\mathrm{p}} f+\frac{1}{2} \frac{\partial}{\partial v_{\mathrm{p}}}\left(\beta v_{\mathrm{p}} f\right)+\frac{1}{4} \gamma f\right. \\
& \left.+D_{\mathrm{RF}} v_{\mathrm{p}} \frac{\partial f}{\partial v_{\mathrm{p}}}\right]
\end{aligned}
$$

where $\alpha, \beta$ and $\gamma$ are the Spitzer collision coefficients [24] and $D_{\mathrm{RF}}$ the RF diffusion coefficient. Under the steadystate condition equation (1), combined with equation (2), is reduced to a first-order ordinary differential equation for $f$ and the solution is readily expressed in terms of one-dimensional velocity integrals to be evaluated numerically.

The specific form of the solution depends on the diffusion coefficient $D_{\mathrm{RF}}$, which is expressed as [23,24]

$$
D_{\mathrm{RF}}=K\left|J_{n-1}\left(\frac{k_{\mathrm{p}} v_{\mathrm{p}}}{\omega_{\mathrm{c}}}\right)+\frac{E_{-}}{E_{+}} J_{n+1}\left(\frac{k_{\mathrm{p}} v_{\mathrm{p}}}{\omega_{\mathrm{c}}}\right)\right|^{2}
$$

For harmonics higher than the fundamental, $D_{\mathrm{RF}}$ has oscillating behaviour due to the presence of Bessel functions of the first kind $J_{n}(x)$ (figure 3 ) and contains parameters pertaining to wave propagation and absorption at the resonance that need to be known. Specifically, $E_{-}\left(E_{+}\right)$are the right (left) handed component of the electric field at the resonance, $k_{\mathrm{p}}$ is the perpendicular wave number and $\omega_{\mathrm{c}}$ the cyclotron frequency of the resonating ion. $K$ is a constant representing the amount of RF absorption. In this work $K$ was varied in equation (3) until a chosen value was matched by the total absorbed power density $P_{\mathrm{RF}}$. The latter was expressed as

$$
P_{\mathrm{RF}}=2 \pi n_{\mathrm{s}} \int \mathrm{d} v_{\mathrm{p}} v_{\mathrm{p}}\left(\frac{m_{\mathrm{s}} v_{\mathrm{p}}^{2}}{2}\right) Q(f)
$$

where the known mass $m_{\mathrm{s}}$ and density $n_{\mathrm{s}}$ of the resonating ion appear. $P_{\mathrm{RF}}$ depends on $K$ in equation (4) through the $Q(f)$ term [23]. The power density absorbed by ${ }^{4} \mathrm{He}$ ions can be estimated to be of the order $\approx 10^{5} \mathrm{~W} \mathrm{~m}^{-3}$ for the scenario under study.

Figure 3 shows the calculated ${ }^{4} \mathrm{He}$ ion distribution assuming $P_{\mathrm{RF}}=500 \mathrm{~kW} \mathrm{~m}^{-3}$ and using cold plasma theory (equations (1.18) and (1.58) of [24]) to calculate the resonance parameters $k_{\mathrm{p}}$ and $E_{-} / E_{+}$from measured values. ${ }^{4} \mathrm{He}$ ions accelerated at the third harmonic resonance develop a rather flat tail in phase space extending to high energies from the NBI injection energy up to a certain cut-off value $E_{\alpha}^{*}$. The latter is determined by the appearance of the first zero in the 


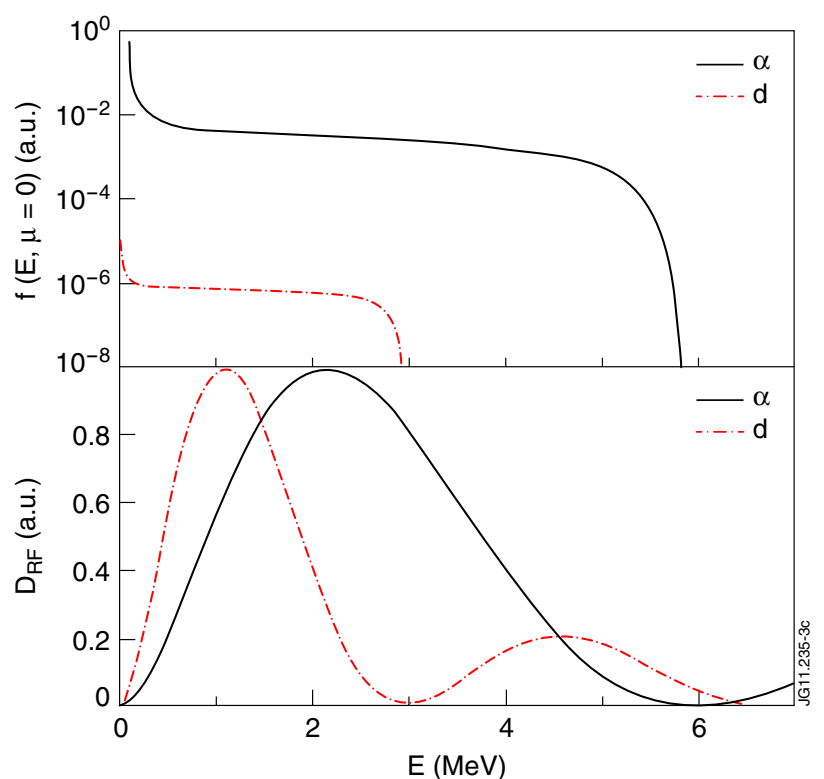

Figure 3. Calculated perpendicular $(\mu=0)$ energy distribution for deuterons and ${ }^{4} \mathrm{He}$ ions accelerated at the third harmonic as described in the text (top) and the corresponding RF diffusion coefficient $D_{\mathrm{RF}}$ (bottom). Coupled power densities

$P_{\mathrm{RF}}=5 \times 10^{5} \mathrm{~W} \mathrm{~m}^{-3}$ and $5 \times 10^{3} \mathrm{~W} \mathrm{~m}^{-3}$ are assumed for ${ }^{4} \mathrm{He}$ ions and deuterons, respectively.

diffusion coefficient $D_{\mathrm{RF}}$ (figure $3(b)$ ). Correspondingly, a sharp drop is seen in the ${ }^{4} \mathrm{He}$ ion energy distribution and almost no particles have energies extending above $E_{\alpha}^{*}$ [24]. Figure 3 also shows the deuteron energy distribution calculated with equation (1), letting $S\left(v_{\mathrm{p}}\right)=L\left(v_{\mathrm{p}}\right)=0$. As ${ }^{4} \mathrm{He}$ ions and deuterons have the same ion cyclotron frequency, part of the incoming wave power is damped on thermal deuterons that also develop energetic tails up to a certain energy $E_{\mathrm{d}}^{*}$. The deuteron tail level is however some orders of magnitude lower compared with that of ${ }^{4} \mathrm{He}$ ions due to the absence of a fast ion seed provided by NBI. As $E_{\mathrm{d}}^{*}$ is also determined by the appearance of the first zero in the diffusion coefficient $D_{\mathrm{RF}}$, the relation $E_{\alpha}^{*}=2 \cdot E_{\mathrm{d}}^{*}$ holds between the two cut-off energies. In fact, $k_{\mathrm{p}}$ and $\omega_{\mathrm{c}}$ being the same for the two species, the cut-off velocity $v_{\mathrm{p}}^{*}$ is the same and the relation between the cut-off energies, $E^{*}=1 / 2 m v^{* 2}$, is given by the mass ratios of the resonating species.

In order to investigate the fractions of RF power absorbed by the different species, simulations were performed with the TOMCAT wave code for single-pass absorption [25]. The ${ }^{4} \mathrm{He}$ ion and deuteron distributions were represented as Maxwellians at temperatures $T_{\alpha} \gg T_{\mathrm{i}}$ and $T_{\mathrm{i}}$, respectively, and a scan was made in ${ }^{4} \mathrm{He}$ temperature and beam density. Figure 4 shows the power fraction absorbed by different species for a case with $T_{\alpha}=50 \mathrm{keV}$ and a beam density equal to $6 \%$ of the electron density. The incoming wave is mainly damped on the ${ }^{4} \mathrm{He}$ beam ions and electrons, with only about $1 \%$ of the power coupled to deuterons. The simulations show quantitative changes depending on the exact value of $T_{\alpha}$ and the beam fraction. However, in all cases only a fraction around $1 \%$ of the power is coupled to deuterons and can be considered independent of the simplistic representation of the ${ }^{4} \mathrm{He}$ ion energy distribution as a Maxwellian at $T_{\alpha} \gg T_{\mathrm{i}}$. Thus, one

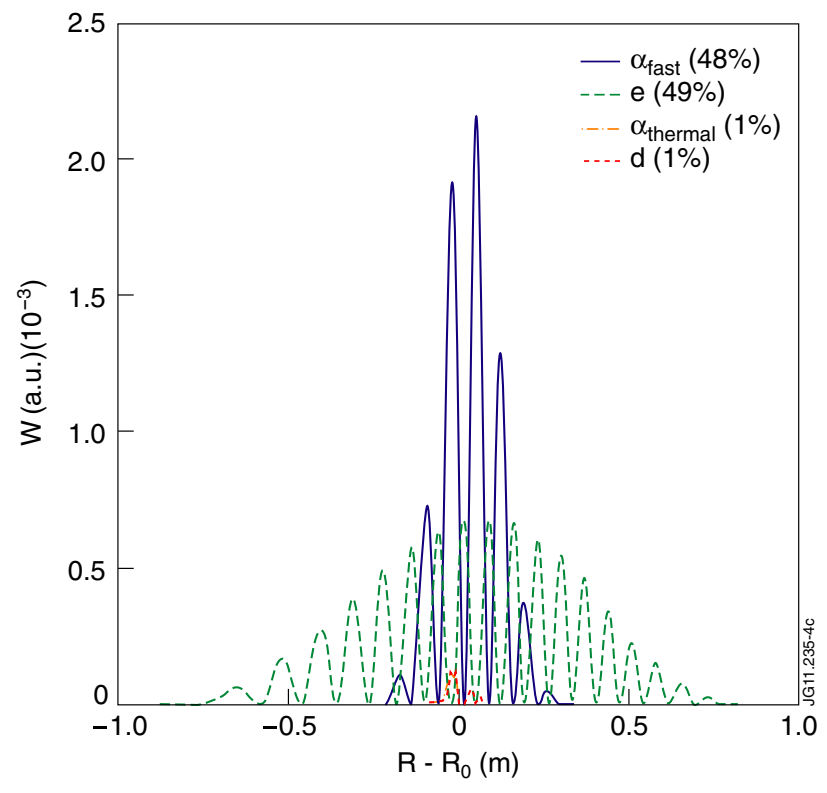

Figure 4. Single-pass wave absorption simulated with the TOMCAT code for a (D) ${ }^{4} \mathrm{He}$ plasma with ${ }^{4} \mathrm{He}$ NBI. The beam was represented in the code as a $T_{\alpha}=50 \mathrm{keV}$ Maxwellian with $6 \%$ concentration. The power fractions absorbed by the different species are written in parentheses.

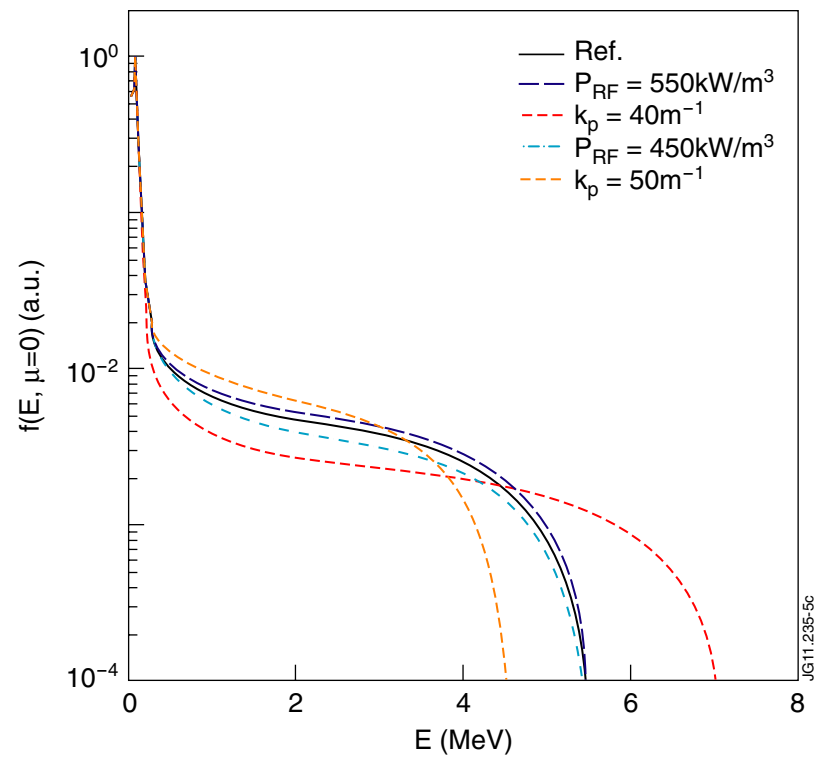

Figure 5. Changes in the ${ }^{4} \mathrm{He}$ ion perpendicular energy distribution by variations of the coupled power density $P_{\mathrm{RF}}$ and perpendicular wave number $k_{\mathrm{p}}$ of $\pm 10 \%$ around reference values.

can expect $P_{\mathrm{RF}}$ to be of the order $\approx 10^{3} \mathrm{~W} \mathrm{~m}^{-3}$ for deuterons at resonance. In figure 3 the value $P_{\mathrm{RF}}=5 \times 10^{3} \mathrm{~W} \mathrm{~m}^{-3}$ was used to calculate the deuteron distribution.

Figure 5 shows variations of the ${ }^{4} \mathrm{He}$ ion distribution by changes of $\pm 10 \%$ in $P_{\mathrm{RF}}$ and $k_{\mathrm{p}}$ around the values predicted by cold wave theory. A small variation in $k_{\mathrm{p}}$ strongly affects the energy distribution, as it directly acts on the cut-off energy $E^{*}$, while $P_{\mathrm{RF}}$ mainly acts on the level of the plateau, without a significant influence on $E^{*}$. Similar trends are seen in the deuteron energy distribution. 


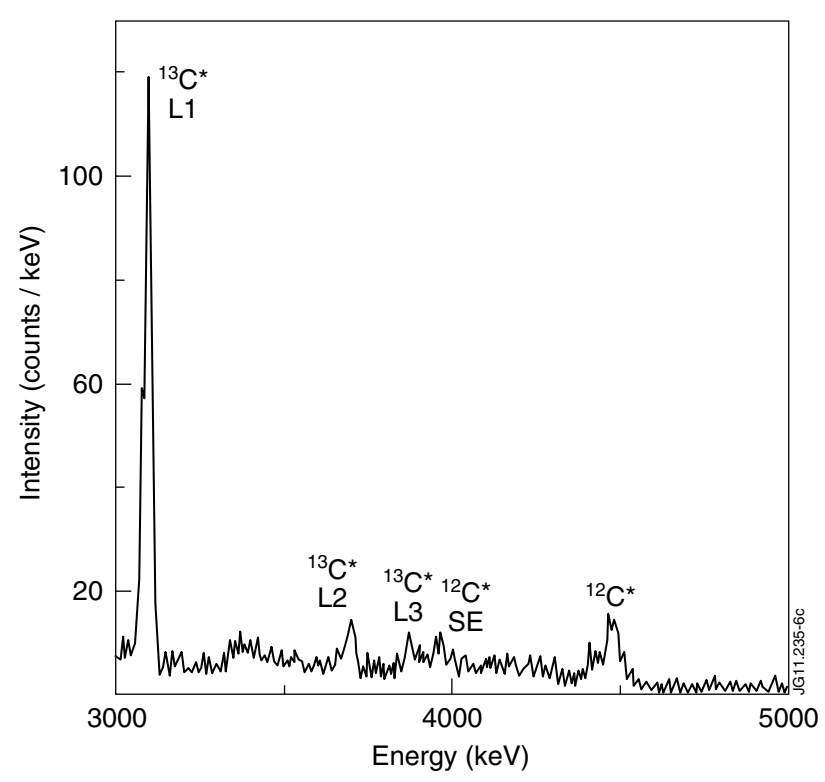

Figure 6. $\gamma$-ray emission spectrum recorded with a $\mathrm{HpGe}$ detector for the sum of JET discharges \#79168-79171 in the time window $12-17 \mathrm{~s}$. Labels indicate peaks from different excited states of ${ }^{13} \mathrm{C}^{*}$ (L1-L3) and ${ }^{12} \mathrm{C}^{*}$, the latter also with its single escape (SE) peak.

\section{Experimental results and modelling}

The measured gamma ray emission spectrum in the region $E_{\gamma}>3 \mathrm{MeV}$ for JET discharges \#79168-79171 is shown in figure 6 in the time window 12-17 s. The spectrum was collected with a HpGe detector, time integrated during RF heating and summed over these four similar discharges to improve statistics. The resolution of the measurement in this energy region is about $1 \mathrm{keV}$, which allows one to appreciate broadening of the peak shapes besides identifying signatures of de-excitation from different states of the same emitting nucleus. The most intense peak appears at $E_{\gamma}=3.09 \mathrm{MeV}$ that corresponds to de-excitation of the first ${ }^{13} \mathrm{C}^{*}$ excited level produced by the $\mathrm{d}+{ }^{12} \mathrm{C}$ reaction. The 3.68 and $3.85 \mathrm{MeV}$ peaks from de-excitation of the second and third ${ }^{13} \mathrm{C}^{*}$ states (the latter barely emerging from the background) are also seen and qualitatively indicate deuteron energies greater than $1.13 \mathrm{MeV}$. ${ }^{4} \mathrm{He}$ ions with energies in excess of $1 \mathrm{MeV}$ are manifested by the appearance of the $E_{\gamma}=4.44 \mathrm{MeV}$ peak. This is in turn mirrored in the single escape peak at $E_{\gamma}=$ $3.93 \mathrm{MeV}$ which depends on the detector response function and does not correspond to a new reaction occurring in the plasma. No clear evidence of the $3.22 \mathrm{MeV}$ peak from de-excitation of the second ${ }^{12} \mathrm{C}^{*}$ excited state is seen in the data.

In addition to qualitatively indicating that ions exceeding a certain energy were produced in the analysed discharges, quantitative information can be derived from the measured spectrum by comparison with modelling. From the experimental data shown in figure 6 one can derive the peak ratio $r$ between counts due to de-excitation of the first and second ${ }^{13} \mathrm{C}^{*}$ levels, yielding $r=6.5 \pm 0.6$ after correcting for the difference in detector intrinsic efficiency at the two peaks and background subtraction. The peak ratio is related to the

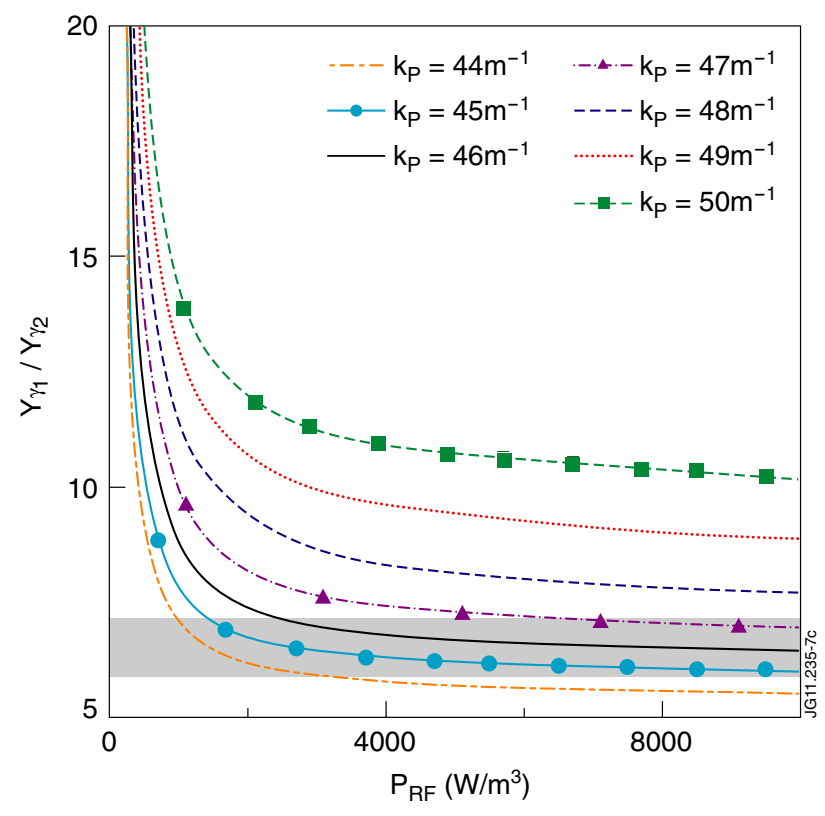

Figure 7. Calculated peak ratio $r=Y_{\gamma_{1}} / Y_{\gamma_{2}}$ between the first and second level of ${ }^{13} \mathrm{C}^{*}$ as a function of the perpendicular wave number $k_{\mathrm{p}}$ and coupled power density $P_{\mathrm{RF}}$ of the deuteron energy distribution. The shadowed bar represents the measured value with its uncertainty.

ratio of the reactivities $Y_{\gamma}$ through the relation [19]

$$
r=\frac{Y_{\gamma 1}}{Y_{\gamma 2}}=\frac{\int \mathrm{d}^{3} \vec{v} \sigma_{\gamma 1} v f(\vec{v})}{\int \mathrm{d}^{3} \vec{v} \sigma_{\gamma 2} v f(\vec{v})}
$$

where $\sigma_{\gamma i}$ represents the cross section for populating the $i$ th ${ }^{13} \mathrm{C}^{*}$ level. The advantage of distinguishing several excited states of the same emitting nucleus is that the calculated peak ratio is independent of the densities of the reactant $\left(\mathrm{d}\right.$ and ${ }^{12} \mathrm{C}$ for the present reaction) and of the effective emission volume, which both are simplified in the ratio expressed by equation (5).

The expected peak ratio was evaluated with equation (5) and is compared with the measured value in figure 7 . The fast deuteron distribution was varied by independently changing the $P_{\mathrm{RF}}$ and $k_{\mathrm{p}}$ parameters and resulted in the calculated curves shown in the figure. The wave polarization was set to a constant value obtained by applying cold plasma theory as it did not significantly affect the distribution. The horizontal shadowed bar represents the experimental value $r$ with its uncertainty. The calculated curves are rather independent of $P_{\mathrm{RF}}$ but for very low power densities, say $P_{\mathrm{RF}}<1 \mathrm{~kW} \mathrm{~m}^{-3}$. Here a sharp rise is seen, which is due to the rapidly decreasing cross section for populating the second ${ }^{13} \mathrm{C}^{*}$ state.

From the results of figure $7, k_{\mathrm{p}}$ must lie in the range $44-47 \mathrm{~m}^{-1}$ that corresponds to $E_{\mathrm{d}}^{*}=3.0 \pm 0.2 \mathrm{MeV}$. This interval is consistent with the estimated $P_{\mathrm{RF}}$ in the range $10^{3}-10^{4} \mathrm{~W} \mathrm{~m}^{-3}$ and reproduces the absolute number of counts measured under the $E_{\gamma}=3.09 \mathrm{MeV}$ and $E_{\gamma}=3.68 \mathrm{MeV}$ peaks assuming ${ }^{12} \mathrm{C}$ concentrations of a few per cent. Lower $k_{\mathrm{p}}$ values are, for instance, ruled out, as the number of counts under the $E_{\gamma}=3.68 \mathrm{MeV}$ peak would be underestimated by 1-2 orders of magnitude. As the electron density varied between $2.3 \times 10^{19}$ and $3.6 \times 10^{19} \mathrm{~m}^{-3}$ in the time window $12-17 \mathrm{~s}$, which changed $k_{\mathrm{p}}$ in time, the value $k_{\mathrm{p}}=44-47 \mathrm{~m}^{-1}$ 


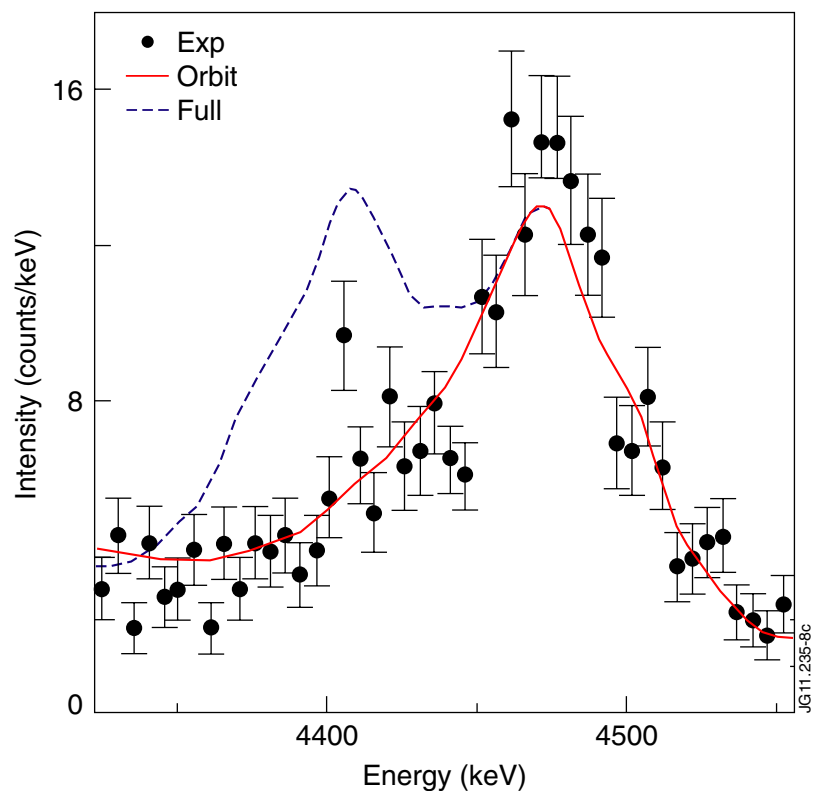

Figure 8. Doppler-broadened $E_{\gamma}=4.44 \mathrm{MeV}$ peak from the ${ }^{9} \mathrm{Be}(\alpha$, $\mathrm{n} \gamma)^{12} \mathrm{C}$ reaction as measured with the $\mathrm{HpGe}$ detector for the sum of JET discharges \#79168-79171 in the time window 12-17 s. The curves are the result of Monte Carlo simulations with the GENESIS code with (solid line) and without (dashed line) correlation between the gyroradius of ${ }^{4} \mathrm{He}$ ions and the width of the line of sight.

must be regarded as effective, i.e. inferred by time integrating the $\gamma$-ray spectra for statistical reasons. Temporal changes in $k_{\mathrm{p}}$ could be resolved in future measurements with better statistics (see discussion). From the relation $E_{\alpha}^{*}=2 \cdot E_{\mathrm{d}}^{*}$ one can also derive the cut-off energy of ${ }^{4} \mathrm{He}$ ions to be $E_{\alpha}^{*}=6.0 \pm 0.4 \mathrm{MeV}$.

We now move to the analysis of the detailed $E_{\gamma}=$ 4.44 $\mathrm{MeV}$ peak shape from the ${ }^{9} \mathrm{Be}(\alpha, \mathrm{n} \gamma){ }^{12} \mathrm{C}$ reaction. Data collected with the $\mathrm{HpGe}$ detector for the sum of four discharges during RF heating are presented in figure 8 . The error bars are those associated with the Poisson statistics of the measurement. The energy calibration is linearly extrapolated from known calibration source peaks at lower energy, with an estimated error of $\approx 3 \mathrm{keV}$ in the region of the $\alpha+{ }^{9} \mathrm{Be}$ peak. Two features are clearly displayed by the data. The first one is that the $\alpha+{ }^{9} \mathrm{Be}$ peak is Doppler broadened, as was found for gamma ray peaks from reactions between RF-heated ${ }^{3} \mathrm{He}$ ions and ${ }^{12} \mathrm{C}$ in [12]. The second is that the centroid of the peak is around $4470 \mathrm{keV}$, i.e. $\approx 30 \mathrm{keV}$ upward shifted with respect to the expected value of $4439 \mathrm{keV}$.

The reason for this shift is clarified when simulating the expected peak shape produced by ${ }^{4} \mathrm{He}$ ions described by the distribution in figure 5. The simulations were performed with the GENESIS Monte Carlo code [12]. The ${ }^{12} \mathrm{C}^{*}$ energy spectrum is determined through classical kinematics by sampling the reactant energy distributions. In a second stage, the resulting gamma ray emission spectrum is evaluated along a specified line of sight. Isotropic $\gamma$-ray emission with respect to the excited nucleus direction in the laboratory frame is assumed and branching ratios are taken into account so as to simulate cascade transitions when necessary [19]. The result of the simulation is presented in figure 8 (dashed line). The only parameter fitted is the normalization of the spectrum with

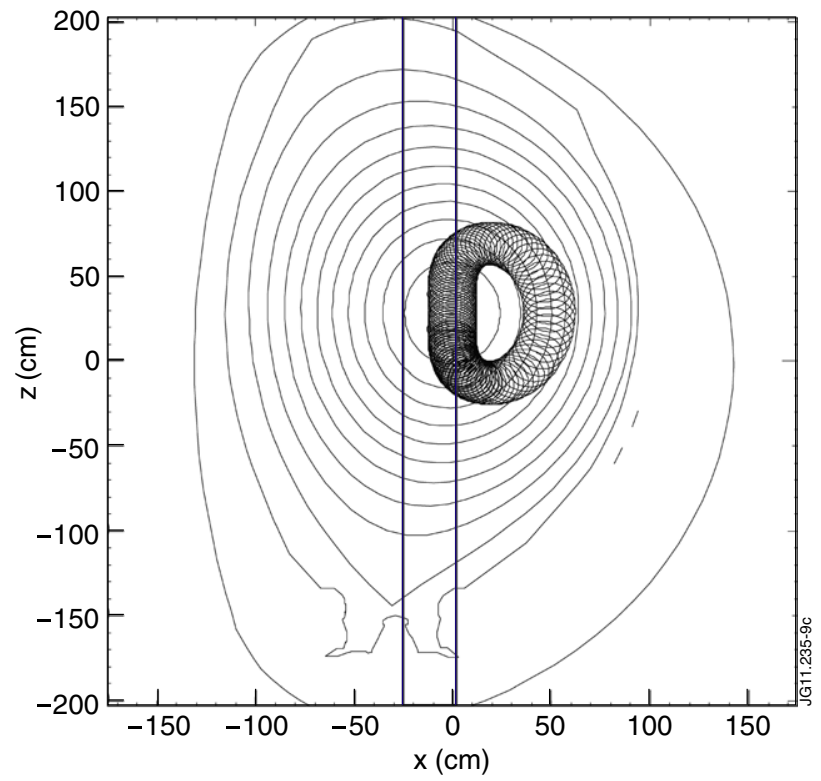

Figure 9. Orbit of a $4 \mathrm{MeV}^{4} \mathrm{He}$ ion projected onto the poloida plane. The particle originates on the magnetic axis $(z=0 \mathrm{~cm}$, $x=0 \mathrm{~cm}$ ) with a pitch angle of $90^{\circ}$. The rectangle represents the detector line of sight.

the addition of a linear background. Quite surprisingly, the curve completely fails to describe the lower half of the peak, which is not present in the measured data. The reason for this effect may be explained by considering the orbit of RF-heated ${ }^{4} \mathrm{He}$ ions at the resonance. The calculated trajectory of $4 \mathrm{MeV}$ ${ }^{4} \mathrm{He}$ ions projected onto the poloidal plane is shown in figure 9 . The particles are generated on the magnetic axis with $90^{\circ}$ pitch angle. The line of sight of the $\mathrm{HpGe}$ detector is determined by collimators designed for the JET Roof Laboratory and is the same as that of the TOFOR neutron spectrometer [26, 27]. The latter was evaluated in [28] and covers the region in major radius $2.74-3.02 \mathrm{~m}$, with an uncertainty of about $1 \mathrm{~cm}$ at the edges. When the line of sight is taken into account in figure 9, it is revealed that only the upward gyro-motion of the particle is seen by the detector. This explains why the lower half of the peak, which corresponds to downward motion, is missing from the data. In order to quantitatively describe the measurements, an 'ad hoc' orbit effect was introduced in the code. This was done by sampling the radial position of the ${ }^{4} \mathrm{He}$ ions with Gaussian probability. The centre of the radial distribution was placed at the resonance location while the FWHM was varied between 5 and $30 \mathrm{~cm}$. At each particle location only the fraction of gyroradius falling inside the line of sight was considered for the calculation of the resulting $\gamma$-ray spectrum. In this way, the measured peak shape is described when the FWHM of the ${ }^{4} \mathrm{He}$ ion radial distribution lies in the range 10 $20 \mathrm{~cm}$ (figure 8 , solid line); here a reduced $\chi^{2} \approx 1.5$ is found, with little variations depending on the exact value of the chosen FWHM in the interval $10-20 \mathrm{~cm}$.

Simulations of the $4.44 \mathrm{MeV}$ peak show changes in shape depending on the $k_{\mathrm{p}}$ parameter of the ${ }^{4} \mathrm{He}$ ion energy distribution. Figure 10 compares the upper half of the 4.44 MeV peak with calculations. The comparison is limited to this part of the peak so as to discard the orbit effect discussed above. The best fit is obtained for $k_{\mathrm{p}} \approx 45 \mathrm{~m}^{-1}$ (reduced 


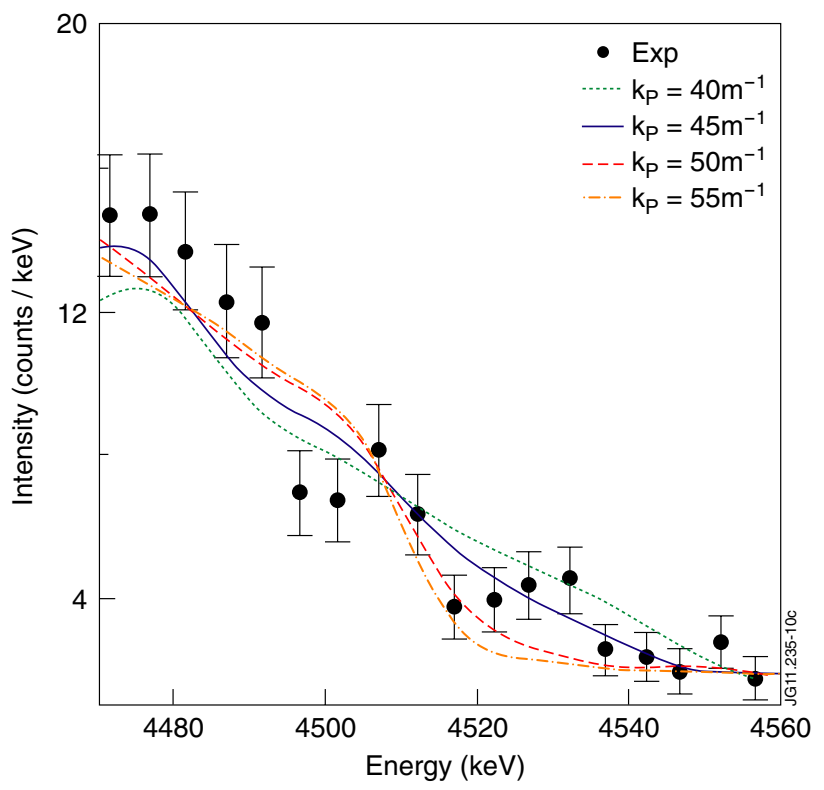

Figure 10. Upper half of the $E_{\gamma}=4.44 \mathrm{MeV}$ peak from the ${ }^{9} \mathrm{Be}(\alpha$, n $)^{12} \mathrm{C}$ reaction and simulations with the GENESIS Monte Carlo code for several values of the perpendicular wave number $k_{\mathrm{p}}$.

$\left.\chi^{2}=1.0\right)$. The range of variation for $k_{\mathrm{p}}$, determined by an increase of \pm 1 in reduced $\chi^{2}$, is $35-55 \mathrm{~m}^{-1}$, which is rather large because of the limited statistics of the measurement. $k_{\mathrm{p}}=45 \pm 10 \mathrm{~m}^{-1}$ is in good agreement with the interval $44-47 \mathrm{~m}^{-1}$ obtained from the analysis of the ${ }^{13} \mathrm{C}^{*}$ peak ratio $r$ and confirms the value $6.0 \pm 0.4 \mathrm{MeV}$ for $E_{\alpha}^{*}$. This result shows that information on the confined ${ }^{4} \mathrm{He}$ energy distribution can indeed be derived from the detailed peak shape for the scenario of this experiment and independently benchmarks the information obtained from the ${ }^{13} \mathrm{C}^{*}$ peak ratio. In figure $10 k_{\mathrm{p}}$ was varied in steps of $5 \mathrm{~m}^{-1}$, but smaller variations may be appreciable in future measurements with improved statistics.

A $\mathrm{LaBr}_{3}(\mathrm{Ce})$ spectrometer was also used in the measurements. The detector, designed for high rates, has already been described in [11]. Although its resolution is not as good as that of $\mathrm{HpGe}$, its enhanced detection efficiency allows for better statistics. Figure 11 shows the measured gamma emission spectrum for JET discharge \#79174. In addition to peaks from the $\mathrm{d}+{ }^{12} \mathrm{C}$ and $\alpha+{ }^{9} \mathrm{Be}$ reaction, a further peak at $E_{\gamma}=3.37 \mathrm{MeV}$ appears. This is produced by the ${ }^{9} \mathrm{Be}(\mathrm{d}, \mathrm{p} \gamma){ }^{10} \mathrm{Be}$ reaction and was not observed in shots \#79168-79171. As the antenna had $\pm 90^{\circ}$ phasing in discharge \#79174 (to be compared with dipole phasing for \#7916879171), cold plasma theory predicts an increased $k_{\mathrm{p}}$, which would correspond to a decreased $E_{\mathrm{d}}^{*}$. In fact, according to figure 7, an augmented $k_{\mathrm{p}}$ is compatible with an increased peak ratio $r$, which for this discharge equals $17 \pm 8$. The large error on this value comes from background subtraction below the $E_{\gamma}=3.68 \mathrm{MeV}$ peak and is partially due to the still not optimized detector parameters adopted in this discharge. As $E_{\mathrm{d}}^{*}$ is lowered, the appearance of the peak from the ${ }^{9} \mathrm{Be}(\mathrm{d}, \mathrm{p} \gamma){ }^{10} \mathrm{Be}$ reaction can be explained only by higher $P_{\mathrm{RF}}$. The temporal evolution of the $E_{\gamma}=3.09 \mathrm{MeV}$ and $E_{\gamma}=4.44 \mathrm{MeV}$ count rates is shown in figure 12 every $20 \mathrm{~ms}$ between 3 and $8 \mathrm{~s}$, together with the corresponding heating pattern and electron density. The count rate starts to rise at

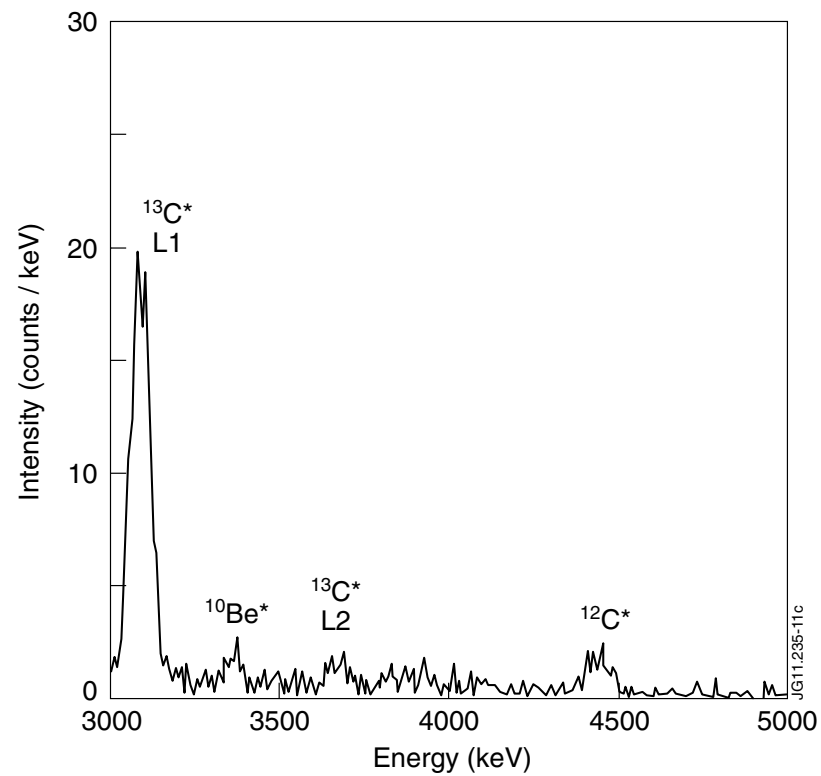

Figure 11. $\gamma$-ray emission spectrum recorded with a $\mathrm{LaBr}_{3}(\mathrm{Ce})$ detector for JET discharge \#79174 in the time window 4-8 s. Shown are peaks from different excited states of ${ }^{13} \mathrm{C}^{*}$ (L1 and L2) as well as peaks from ${ }^{12} \mathrm{C}^{*}$ and ${ }^{10} \mathrm{Be}^{*}$.

$4 \mathrm{~s}$ and drops between 7.2 and 7.6s, i.e. $0.4 \mathrm{~s}$ before the end of the RF heating phase, between 4 and $8 \mathrm{~s}$ in this discharge. The reason is that the core electron density experiences a jump from $3 \times 10^{19} \mathrm{~m}^{-3}$ at $7 \mathrm{~s}$ to $3.8 \times 10^{19} \mathrm{~m}^{-3}$ at $7.2 \mathrm{~s}$ that impedes fast ion acceleration, with a delayed drop in the $\gamma$ count rate due to slowing down. Between 5.6 and $5.8 \mathrm{~s}$ a further sharp drop of a factor $\approx 1.6$ that exceeds the statistical fluctuation of the data is observed in the $E_{\gamma}=3.09 \mathrm{MeV}$ count rate. This drop corresponds to the appearance of toroidal Alfvén eigenmodes (TAE) in the magnetic traces (figure 13) at $5.75 \mathrm{~s}$ and suggests redistribution of fast deuterons. A similar drop is not observed in the count rate for the $E_{\gamma}=4.44 \mathrm{MeV}$ peak $\left(\alpha+{ }^{9} \mathrm{Be}\right.$ reaction) but might be masked by the larger statistical data fluctuation. No signs of MHD activity was observed in the magnetic traces, as well as in the $\gamma$-ray count rates, for shots \#79168-79171 where the HpGe detector was used.

\section{Discussion}

$\gamma$-ray observations can be compared with neutron measurements with the TOFOR spectrometer on JET [26,27]. The instrument measures the neutron time of flight $t_{\mathrm{n}}$ between two arrays of scintillators so that the neutron energy $E_{\mathrm{n}}$ is univocally related to $t_{\mathrm{n}}$ through $E_{\mathrm{n}}=1.04 \times 10^{4} / t_{\mathrm{n}}^{2}$ where $E_{\mathrm{n}}$ is expressed in $\mathrm{MeV}$ and $t_{\mathrm{n}}$ in nanoseconds. This relation implies that higher neutron energies correspond to smaller time of flights. Figure 14 shows the measured neutron spectrum for the sum of JET discharges \#79168-79171 in the time window $12-17 \mathrm{~s}$ that directly compares with the $\gamma$-ray spectrum of figure 6. Neutron emission is expected mainly from the $\mathrm{d}+\mathrm{d} \rightarrow \mathrm{n}+{ }^{3} \mathrm{He}$ reaction, but there is a component from the $\alpha+{ }^{9} \mathrm{Be} \rightarrow \mathrm{n}+{ }^{12} \mathrm{C}$ reaction as well. Noticeably, the highly non-Maxwellian character of the energy distribution is manifested in the neutron spectrum that does not show a Gaussian 


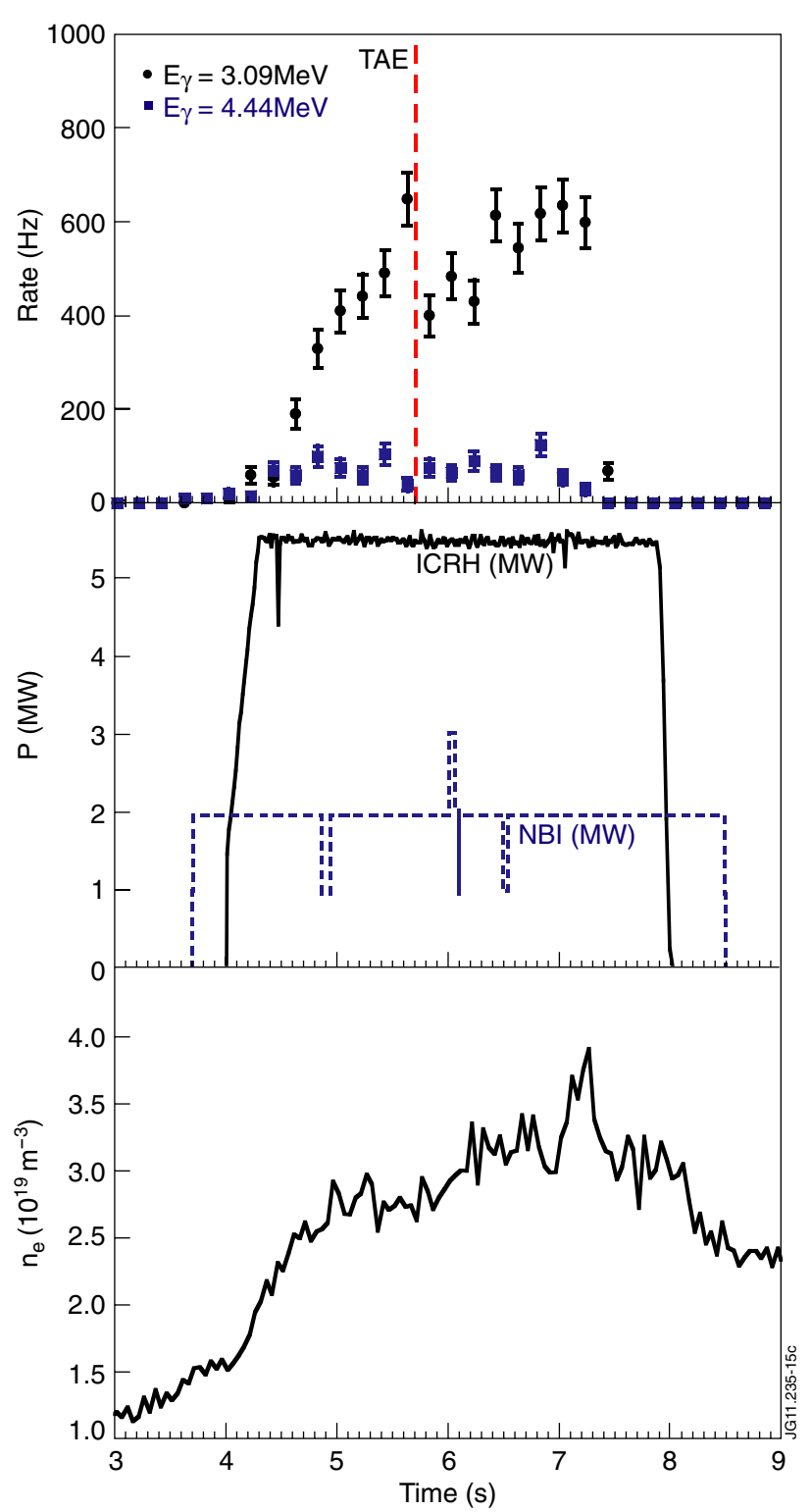

Figure 12. (a) Count rates of the $E_{\gamma}=3.09 \mathrm{MeV}$ and $E_{\gamma}=4.44 \mathrm{MeV}$ peaks from the ${ }^{12} \mathrm{C}(\mathrm{d}, \mathrm{p} \gamma){ }^{13} \mathrm{C}$ and ${ }^{9} \mathrm{Be}(\alpha, \mathrm{n} \gamma){ }^{12} \mathrm{C}$ reactions measured for discharge \#79174. The vertical dashed line represents the instant when a TAE was detected in the magnetic traces. The applied heating pattern and electron density evolution in the time window $3-9 \mathrm{~s}$ are shown in $(b)$ and $(c)$, respectively.

peak centred at $t_{\mathrm{n}}=65 \mathrm{~ns}\left(E_{\mathrm{n}}=2.45 \mathrm{MeV}\right)$ due to thermal emission. The GENESIS code was used to calculate the expected neutron spectrum from the $\mathrm{d}+\mathrm{d} \rightarrow \mathrm{n}+{ }^{3} \mathrm{He}$ and $\alpha+{ }^{9} \mathrm{Be} \rightarrow \mathrm{n}+{ }^{12} \mathrm{C}$ reactions using as input the distributions calculated as described in section 3 and setting $k_{\mathrm{p}}$ to the value $45 \mathrm{~m}^{-1}$ inferred from $\gamma$-ray measurements. As the result of equation (2) is not adequate in the thermal region, the deuteron distribution was joined here to an isotropic Maxwellian representing the bulk component with a procedure similar to that adopted in [29]. This choice, although simplistic, was found adequate to describe the data within the statistics. The same procedure was not necessary for the neutron component from the $\alpha+{ }^{9} \mathrm{Be}$ reaction, as neutron emission for thermal ${ }^{4} \mathrm{He}$ ions is negligible. The result of the simulation is shown in

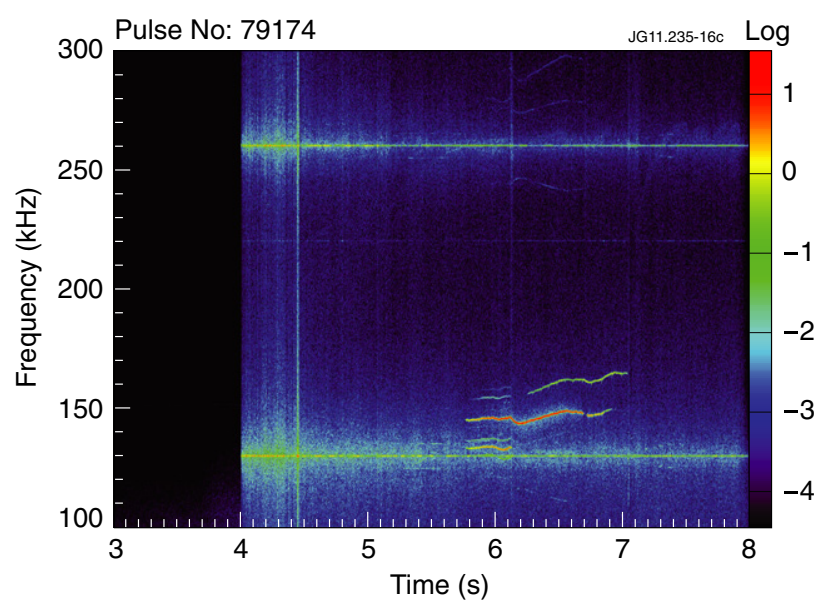

Figure 13. Frequency spectrogram from Mirnov coils showing TAEs in the frequency range $100-200 \mathrm{kHz}$ for discharge \#79174.
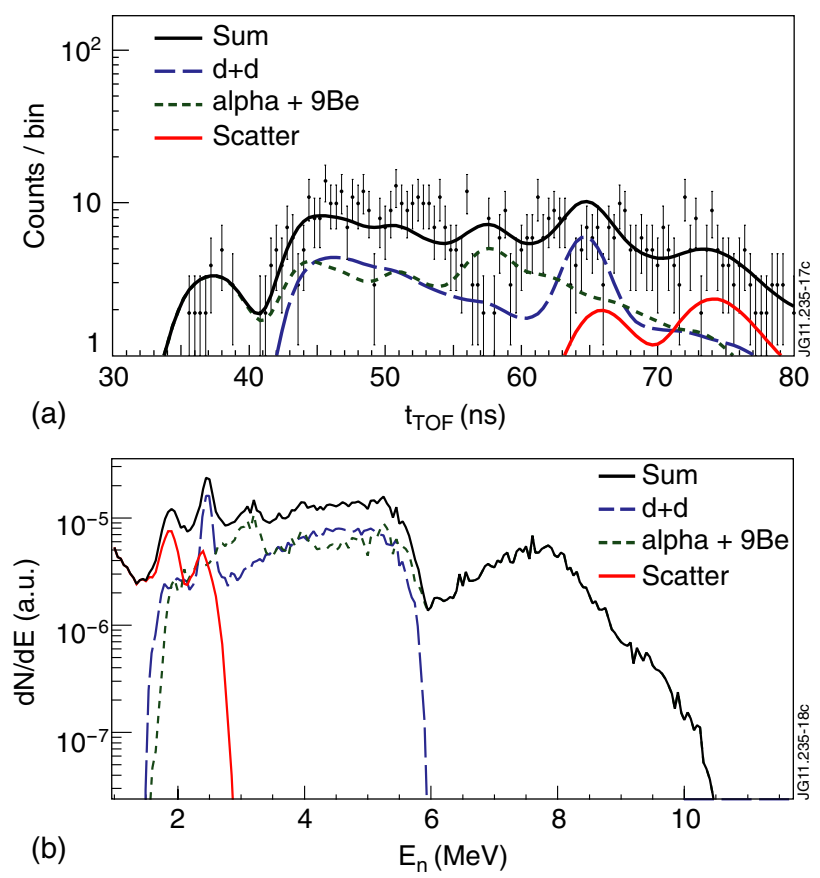

Figure 14. Neutron spectrum measured with the TOFOR spectrometer for the sum of JET discharges \#79168-79171 in the time interval $12-17 \mathrm{~s}$. The curves are simulations of the expected $\mathrm{d}+\mathrm{d} \rightarrow \mathrm{n}+{ }^{3} \mathrm{He}$ (long dashed) and $\alpha+{ }^{9} \mathrm{Be} \rightarrow \mathrm{n}+{ }^{12} \mathrm{C}$ (short dashed) neutron spectra, plus a scatter component (red solid) taking into account neutron diffusion from the tokamak walls. Both the measured time of flight spectrum $(a)$ and the corresponding best fit neutron energy spectrum $(b)$ are shown in the figure.

figure 14 and confirms the presence of ${ }^{4} \mathrm{He}$ ions with energies as high as $6 \mathrm{MeV}$ determined through $\gamma$-ray spectroscopy. It is also revealed that the $\mathrm{d}+\mathrm{d}$ contribution has a sharp cutoff at $t_{\mathrm{n}}=42 \mathrm{~ns}$ which sets $E_{\mathrm{d}}^{*} \approx 3 \mathrm{MeV}$, consistent with $\gamma$ observations. More details on neutron measurements for this experiment can be found in [30]. Here we just add that orbit effects need to be taken into account to describe neutron data, for which the empirical model illustrated in section 3 was adopted in the simulations of figure 14. A more accurate procedure was used in [30] and its developments are the subject of [31,32]. A similar study of the energy distribution of fast deuterons 
accelerated by RF at the third harmonic using neutron emission spectroscopy at JET is reported in [33] for a different experiment and confirms the results presented here. In [34] measurements with a Faraday cup array are reported for the experiment considered here. A signal in terms of current from ${ }^{4} \mathrm{He}$ ions in the energy range $2.3-5.9 \mathrm{MeV}$ was detected for the first time throughout all the discharges, while no evidence of ${ }^{4} \mathrm{He}$ ions exceeding $6 \mathrm{MeV}$ was seen. This is also consistent with the values stated in this paper.

In this work we have derived information on energy distribution of ${ }^{4} \mathrm{He}$ ions by considering both variations in the ratio of peaks produced by different excited states of the same emitting nucleus $\left({ }^{13} \mathrm{C}^{*}\right.$ in this case $)$ and by studying the detailed shape of the $\alpha+{ }^{9} \mathrm{Be}$ peak at $E_{\gamma}=4.44 \mathrm{MeV}$. Although qualitative results could be derived by simple arguments based on the observed emission spectra, quantitative information was obtained only by considering the detailed nuclear physics behind the reaction in terms of differential cross section, energy levels and branching ratios of the emitting nuclei, which can be quite different depending on the emission process. In fact, the extremely varied properties of reactions between light nuclei and impurities (see for example [35, 36] and references therein) can sometimes produce undesirable effects, such as limiting changes of an observable even over significant modifications in the plasma parameters. An example is given by the peak ratio of the ${ }^{12} \mathrm{C}\left({ }^{3} \mathrm{He}, \mathrm{p} \gamma\right){ }^{14} \mathrm{~N}$ reaction, which is rather independent of the fast ${ }^{3} \mathrm{He}$ distribution as shown in [19], in clear contrast with the peak ratio from the ${ }^{12} \mathrm{C}(\mathrm{d}, \mathrm{p} \gamma){ }^{13} \mathrm{C}$ reaction considered in this paper. Similar arguments hold for the peak shape that is found to depend on the detailed angular distribution of the emitting nuclei and can also show limited variations for certain reactions. These issues will be more extensively addressed in forthcoming papers. Clearly, due to the Poissonian nature of the process, the possibility to observe changes in the $\gamma$-ray emission spectrum benefits from increased statistics which sets a trade-off between time resolution and the required precision in determining the parameter of interest (for example $E_{\alpha}^{*}$ in this paper). To allow for a quantitative study, the time resolution of the measurements presented in this paper was limited, especially for observations with the $\mathrm{HpGe}$ detector, where four similar discharges had to be summed to achieve sufficient statistics. This limitation is, however, of no particular relevance for similar measurements on ITER, thanks to significantly increased $\gamma$-ray fluxes. A calculation of the expected $\gamma$-ray counting rates for a ITER DT plasma reveals that rates in the $\mathrm{MHz}$ range can be expected from the ${ }^{9} \mathrm{Be}(\alpha, \mathrm{n} \gamma){ }^{12} \mathrm{C}$ reaction for a $\mathrm{LaBr}_{3}(\mathrm{Ce})$ detector placed in the ITER vertical camera [37]. This would translate into counting rates in the $100 \mathrm{kHz}$ range for $\mathrm{HpGe}$ detectors of the same dimensions, due to their lower efficiency with respect to $\mathrm{LaBr}_{3}(\mathrm{Ce})$, still allowing measurements with a time resolution of some milliseconds. A crucial issue for $\gamma$-ray measurements on ITER is instead the operation in a high neutron background, which is a reason of concern especially for $\mathrm{HpGe}$, as performance degrading neutron damage is significantly more pronounced for this detector. A promising solution to this problem is the use of ad hoc designed attenuators, such as $\mathrm{LiH}$ [37], and will be extensively tested in experiments at nuclear accelerators and in the future JET deuterium-tritium campaign with an ITER-like wall.

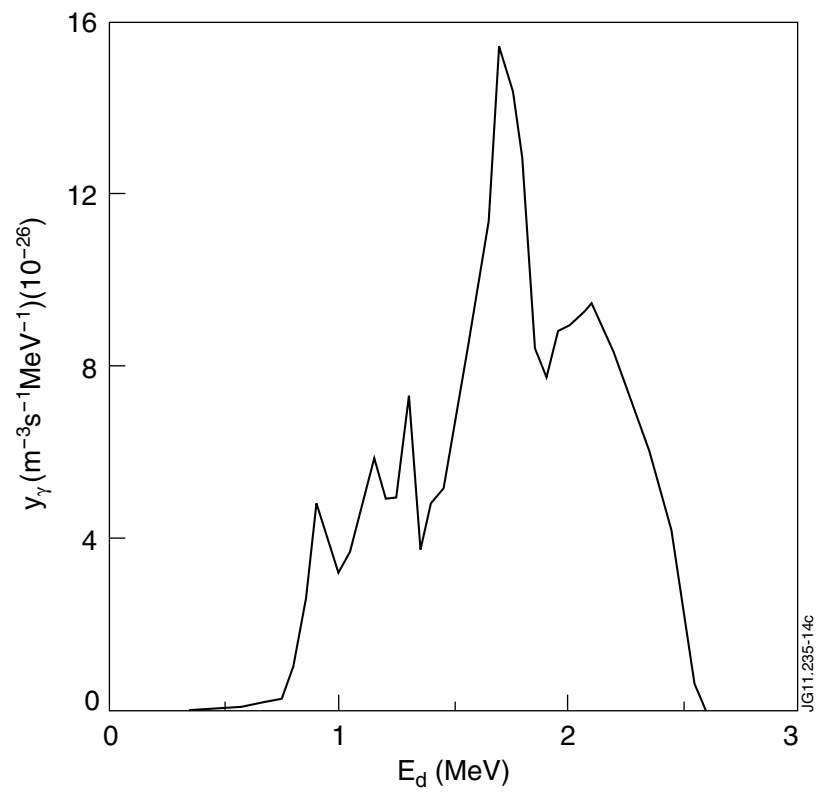

Figure 15. Differential reactivity for the production of $E_{\gamma}=3.09 \mathrm{MeV} \gamma$-rays from the ${ }^{12} \mathrm{C}(\mathrm{d}, \mathrm{p} \gamma)^{13} \mathrm{C}$ reaction for third harmonic RF heating and parameters described in the text.

Similarly to neutron spectroscopy $[38,39]$, as shown in the result of figure 12, $\gamma$-ray spectroscopy can also be used to derive information on the effect of MHD modes on fast ions by studying the time dependence of the measured counting rate [38]. The energies of the interacting ions are related to the counting rate through the differential reactivity $y_{\gamma}(E)=$ $v \sigma(E) f(E)$. The latter represents the $\gamma$-ray emission intensity as a function of the fast ion energy and is the integrand appearing in equation (5). The differential reactivity for the $E_{\gamma}=3.09 \mathrm{MeV}$ peak of the ${ }^{12} \mathrm{C}(\mathrm{d}, \mathrm{p}){ }^{13} \mathrm{C}$ reaction is shown in figure 15 for discharge \#79174. The curve is significantly non-null only in the energy range $E_{\mathrm{d}}=0.8-2.5 \mathrm{MeV}$, with a peak at $E_{\mathrm{d}} \approx 1.7 \mathrm{MeV}$. By inspecting figure 15 , we speculate that a redistribution of fast deuterons with $E_{\mathrm{d}} \approx 1.7 \mathrm{MeV}$ (i.e. around the peak in the differential reactivity curve) must have occurred in discharge \#79174 to justify a factor 1.6 drop as observed in the counting rate. Further insights into modeparticle interaction could be obtained by calculating resonance curves between the observed TAEs and fast ions, as done for instance in [40], but this goes outside the scope of this paper. Here we note the following fact. The fast ion energies, to which $\gamma$-ray spectroscopy is sensitive, are determined by the differential reactivity of each specific reaction, which comprises the combined product of the cross section and the ion energy distribution. When resonances in the cross sections appear $[35,36]$, the evolution of the $\gamma$-ray counting rate can be dominated by ions with energies near resonance, despite those with energies off-resonance. For example, in the case of figure 15 , a change in the fast deuteron population having $E_{\mathrm{d}} \approx 0.8 \mathrm{MeV}$ would hardly be manifested in the observed $\gamma$-ray counting rate. This strengthens the need to consider the detailed nuclear physics behind each reaction to derive conclusions also on the fast ion dynamics based on the observed $\gamma$-ray emission rate. From the instrumental point of view, observations on the interaction between fast ions and instabilities through $\gamma$ spectroscopy would be boosted by the 
capability to perform spatially resolved measurements, for example to study the effects of $\alpha$ particles in a DT plasma. This could be done through a tomographic system, such as the JET $\gamma$-ray camera [10], but with spectroscopy capabilities at each channel and an increased number of lines of sight. Concept studies are currently underway in view of ITER and some solutions are reported in [37], where the use of a set of $\mathrm{LaBr}_{3}(\mathrm{Ce})$ detectors is considered, the latter being more resilient to neutron damage.

\section{Conclusions}

In this paper first high-resolution measurements of Dopplerbroadened peak shapes from $\gamma$ emitting reactions induced by energetic $\alpha$ particles and deuterons were reported. The data were interpreted through Monte Carlo simulations of the $\gamma$-ray emitting reactions and good agreement was found with measured data. Information on the ${ }^{4} \mathrm{He}$ and deuteron energy distribution was inferred and found consistent with observations from other diagnostics. In particular, the confinement of deuteron and ${ }^{4} \mathrm{He}$ ions with energies as high as $3 \mathrm{MeV}$ and $6 \mathrm{MeV}$, respectively, was assessed. A drop in the $\gamma$-ray count rate was found in one discharge and corresponded to the appearance of TAE in the magnetic traces. The need to consider the detailed nuclear reaction data and physics of each reaction to derive quantitative information on energetic ions from the $\gamma$-ray emission spectrum was pointed out. The methods and results reported here are of relevance for confined fast ion studies in the $\mathrm{MeV}$ range on next generation devices, such as $\alpha$ particles in ITER DT plasmas, where observations would significantly be boosted by spatially resolved measurements through a tomographic $\gamma$-ray system with spectroscopic capabilities.

\section{Acknowledgments}

This work was supported by EURATOM and carried out within the framework of the European Fusion Development Agreement. The views and opinions expressed herein do not necessarily reflect those of the European Commission.

(C) Euratom 2012.

\section{References}

[1] Breizman B.N. and Sharapov S.E. 2011 Plasma Phys. Control. Fusion $\mathbf{5 3} 054001$

[2] García-Muñoz M., Fahrbach H.U., Günter S., Igochine V., Mantsinen M.J., Maraschek M., Martin P., Piovesan P., Sassenberg K. and Zohm H. 2008 Phys. Rev. Lett. 100055005

[3] García-Muñoz M. et al 2010 Phys. Rev. Lett. 104185002

[4] Heidbrink W.W. 2010 Rev. Sci. Instrum. 81 10D727

[5] Heidbrink W.W. et al 2007 Phys. Rev. Lett. 99245002

[6] White R.B., Gorelenkov N., Heidbrink W.W. and Van Zeeland M.A. 2010 Phys. Plasmas 17056107

[7] Donné A.J.H. et al 2007 Nucl. Fusion 47 S337

[8] Källne J., Ballabio L., Frenje J., Conroy S., Ericsson G., Tardocchi M., Traneus E. and Gorini G. 2000 Phys. Rev. Lett. 851246
[9] Korotkov A.A., Gondhalekar A. and Akers R.J. 2000 Phys. Plasmas 7957

[10] Kiptily V.G., Cecil F.E. and Medley S.S. 2006 Plasma Phys. Control. Fusion 48 R59

[11] Nocente M. et al 2010 Rev. Sci. Instrum. 81 10D321

[12] Tardocchi M. et al 2011 Phys. Rev. Lett. 107205002

[13] Mantsinen M.J. et al 2002 Phys. Rev. Lett. 88105002

[14] Ballabio L. 2003 Calculation and measurement of the neutron emission spectrum due to thermonuclear and higher-order reactions in tokamak plasmas $P h D$ Thesis Acta Universitatis Upsaliensis No 797, Faculty of Science and Technology, Uppsala University

[15] Ballabio L., Gorini G. and Källne J. 1997 Phys. Rev. E $\mathbf{5 5} 3358$

[16] Kiptily V.G. et al 2002 Nucl. Fusion 42999

[17] Kiptily V.G. et al 1990 Fusion Technol. 18583

[18] Kiptily V.G. et al 1992 Fusion Technol. 22454

[19] Proverbio I., Nocente M., Kiptily V.G., Tardocchi M. and Gorini G. 2010 Rev. Sci. Instrum. 81 10D320

[20] Kiptily V.G., Matukov A.V., Mishin A.S., Najdenov V.O., Polunovskij I.A., Rassadin L.A. and Chugunov I.N. 1992 Bull Russ. Acad. Sci. Phys. 56125

[21] Van Der Zwan L. and Geiger K.W. 1970 Nucl. Phys. A 152481

[22] Papillon F. and Walter P. 1997 Nucl. Instrum. Methods B 132468

[23] Salmi A., Mantsinen M.J., Beaumont P., De Vries P., Eriksson L.-G., Gowers C., Helander P., Laxåback M., Noterdaeme J.-M. and Testa D. 2006 Plasma Phys. Control. Fusion 48717

[24] Stix T.H. 1992 Waves in Plasmas (New York: Springer)

[25] Van Eester D. and Koch R. 1998 Plasma Phys. Control. Fusion 401949

[26] Gorini G. and Källne J. 1992 Rev. Sci. Instrum. 634548

[27] Gatu Johnson M. et al 2008 Nucl. Instrum. Methods A 591417

[28] Gatu Johnson M. et al 2010 Plasma Phys. Control. Fusion 52085002

[29] Nocente M., Gorini G., Källne J. and Tardocchi M. 2011 Nucl. Fusion 51063011

[30] Gatu Johnson M. et al 2010 Rev. Sci. Instrum. $8110 \mathrm{D} 336$

[31] Eriksson J. et al 2011 Finite Larmor radii effects in fast ion measurements as demonstrated using neutron emission spectrometry of JET plasmas heated with 3rd harmonic ICRF Proc. 38th EPS Conf. on Plasma Physics and Controlled Fusion (Strasbourg, France, 27th June-1st July 2011) http://ocs.ciemat.es/EPS2011PAP/pdf/P2.049.pdf

[32] Eriksson J. et al 2012 Investigation and modelling of finite Larmor radii effects in fast ion measurements with neutron emission spectrometry Plasma Phys. Control. Fusion submitted

[33] Hellesen C. et al 2012 Fast ion distributions from 3rd harmonic ICRF heating studied with neutron emission spectroscopy Nucl. Fusion submitted

[34] Darrow D.S., Cecil F.E., Kiptily V., Fullard K., Horton A. and Murari A. 2010 Rev. Sci. Instrum. 81 10D330

[35] Ajzenberg-Selove F. 1990 Nucl. Phys. A 5061

[36] Ajzenberg-Selove F. 1991 Nucl. Phys. A 5231

[37] Chugunov I.N., Shevelev A.E., Gin D.B., Kiptily V.G., Gorini G., Nocente M., Tardocchi M., Doinikov D.N., Naidenov V.O. and Khilkevitch E.M. 2011 Nucl. Fusion 51083010

[38] Hellesen C. et al 2010 Nucl. Fusion 50022001

[39] Hellesen C. et al 2010 Nucl. Fusion 50084006

[40] Nocente M. et al 2012 Gamma-ray spectroscopy measurements of confined fast ions on ASDEX Upgrade Nucl. Fusion at press 